\title{
Iminosugar idoBR1 Isolated from Cucumber Cucumis sativus Reduces Inflammatory Activity
}

\author{
Robert J. Nash,* Barbara Bartholomew, Yana B. Penkova, Dino Rotondo, Fernanda Yamasaka, \\ Graham P. Stafford, Sarah F. Jenkinson, and George W. J. Fleet
}

Cite This: ACS Omega 2020, 5, 16263-16271

Read Online

\section{ACCESS \\ Џlll Metrics \& More \\ Article Recommendations \\ Supporting Information}

ABSTRACT: Cucumbers have been anecdotally claimed to have anti-inflammatory activity for a long time, but the active principle was not identified. idoBR1, $(2 R, 3 R, 4 R, 5 S)-3,4,5$-trihydroxypiperidine-2-carboxylic acid, is an iminosugar amino acid isolated from fruits of certain cucumbers, Cucumis sativus (Cucurbitaceae). It has no chromophore and analytically behaves like an amino acid making detection and identification difficult. It has antiinflammatory activity reducing lipopolysaccharide (LPS)-induced tumor necrosis factor alpha (TNF- $\alpha$ ) in THP-1 cells and ex vivo human blood. It showed selective inhibition of human $\alpha$-Liduronidase and sialidases from both bacteria (Tannerella forsythia) and human THP-1 cells. idoBR1 and cucumber extract reduced the binding of hyaluronic acid (HA) to CD44 in LPS-stimulated

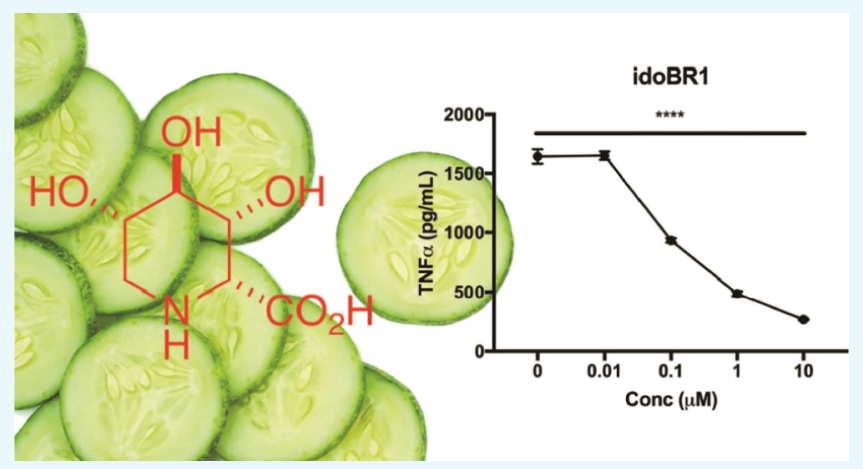
THP-1 cells and may function as an anti-inflammatory agent by inhibiting induced sialidase involved in the production of functionally active HA adhesive CD44. Similar to the related iminosugars, idoBR1 is excreted unchanged in urine following consumption. Its importance in the diet should be further evaluated.

\section{INTRODUCTION}

Cucumber (Cucumis sativus) is a widely cultivated plant in the gourd family Cucurbitaceae, which includes squash. Cucumbers probably originated in India and have been cultivated for at least 3000 years in Western Asia ${ }^{1}$ and probably introduced to other parts of Europe by the Romans. ${ }^{2}$ Records of cucumber cultivation appear in France in the 9th century, in England in the 14th century, and in North America by the mid-16th century.

Cucumbers and cucumber extracts have long been recognized as having anti-inflammatory properties and have been used topically for various types of skin problems, including swelling under the eyes and sunburns. ${ }^{3}$ Cucumber was very popular in the ancient civilizations of Egypt, Greece, and Rome, where it was used not only as a food but also for its skin-healing properties. It has long been recognized by women for benefits to red, tired, and puffy eyes. There are a number of reports of the anti-inflammatory activity of cucumber aqueous extracts, but the active components were not identified; ${ }^{4,5}$ a recent report identified an amino acid fraction as reducing angiotensin II-induced inflammation in human endothelial cells, but only common amino acids could be identified by the liquid chromatography-mass spectrometry (LCMS) method used. $^{6}$ Certain varieties of cucumber have been shown to contain a single iminosugar amino acid, $(2 R, 3 R, 4 R, 5 S)-3,4,5$ trihydroxypiperidine-2-carboxylic acid (idoBR1), a trihydrox- ypipecolic acid (1) (Figure 1). ${ }^{7}$ While many iminosugars have been isolated from plants, BR1 $(2 S, 3 R, 4 R, 5 S)$-3,4,5-trihydrox-

${ }_{1}$

Figure 1. Structures of iminosugar acids idoBR1 (1), BR1 (2), and equivalent iminosugar DNJ (3).

ypiperidine-2-carboxylic acid (2) from Baphia racemosa ${ }^{8,9}$ is one of the very few iminosugar acids that have been identified as a natural product. ${ }^{10}$ Iminosugar acids are much more difficult to isolate and identify than their iminosugar analogues because of the high content of acidic and neutral amino acids in plants. idoBR1 was synthesized before it was isolated. ${ }^{11}$ idoBR1 is reported to have anti-inflammatory activity, and cucumber extract containing idoBR1 has shown benefits in

Received: May 6, 2020

Accepted: June 11, 2020

Published: June 23, 2020 
osteo-arthritis ${ }^{7,12}$ without signs of toxicity. ${ }^{13}$ In the present study, we sought to investigate the anti-inflammatory effects and possible mechanism for idoBR1 and a cucumber extract standardized for the idoBR1 content, Q-actin. BR1 is reported to be a glycosidase inhibitor, ${ }^{8}$ and for the possible mechanism, we decided to focus on glycosidase activity of idoBR1 and, in particular, specific properties/signaling pathways that might be related to the structural similarity of uronic acids.

\section{MATERIALS AND METHODS}

Chemicals. idoBR1 was synthesized ${ }^{11}$ (MI164687 purchased from Carbosynth Limited, Compton, UK). Q-actin idoBR1-standardized cucumber extract (batch no. B17CF007) was obtained from IminoTech Inc, Carson City, Nevada, USA. BR1, 1-deoxynojirimycin (DNJ), and castanospermine were authentic samples from PhytoQuest Limited, Aberystwyth, Iminosugar library. Dexamethasone (DM) and mifepristone were from Sigma-Aldrich.

Gas Chromatography-Mass Spectrometry. Gas chromatography (GC) was found to give better resolution of idoBR1 in extracts than HPLC and allowed easier quantification. Castanospermine was added to the dissolved cucumber extract at $0.2 \mathrm{mg}$ per $\mathrm{g}$ to act as an internal reference. Samples $(0.1 \mathrm{mg})$ of dried cucumber extract or pure compounds were reacted with $30 \mu \mathrm{L}$ of Pierce TriSil reagent; after $20 \mathrm{~min}$, the trimethylsilylated (TMS) compounds were analyzed on a Perkin Elmer Turbomass spectrometer using a high-polarity fused-silica column (Varian "Factor Four" VF$5 \mathrm{~ms}$ column, $25 \mathrm{~m} \times 0.25 \mathrm{~mm}$ i.d., $0.25 \mu \mathrm{m}$ phase thickness). The carrier gas (helium) flow rate was $1 \mathrm{~mL} / \mathrm{min}$. The TMS derivatives were separated using a temperature program starting at $160{ }^{\circ} \mathrm{C}$ for $5 \mathrm{~min}$, followed by a linear increase to $300{ }^{\circ} \mathrm{C}$ at a rate of $10{ }^{\circ} \mathrm{C} / \mathrm{min}$. Electron impact mass spectrometry (MS) of the column eluent was carried out with the quadrupole ion filter system run at $250{ }^{\circ} \mathrm{C}$ constantly during analysis.

TNF- $\alpha$ ELISA on Human Blood. Whole blood was supplied by the Scottish National Blood Transfusion Service (SNBTS), Glasgow, UK, and was preincubated for $48 \mathrm{~h}$ with varying concentrations of Q-actin or idoBR1, after which lipopolysaccharide (LPS) $(10 \mu \mathrm{g} / \mathrm{mL})$ was added, and incubations continued for a further $20 \mathrm{~h}$. Following incubation at $37{ }^{\circ} \mathrm{C}\left(5 \% \mathrm{CO}_{2}, 100 \%\right.$ humidity $)$, plasma was collected from the blood by centrifugation ( $30 \mathrm{~s} 10,000 \mathrm{~g}$ ), and the levels of tumor necrosis factor alpha (TNF- $\alpha$ ) in plasma samples were measured by enzyme-linked immunosorbent assay (ELISA). Values represent the mean \pm sd of $n=3$.

Statistical Analysis. All statistical analyses were performed with GraphPad Prism. Significance is denoted as $*(p=\leq 0.05)$.

Effect of Mifepristone on the idoBR1- and DMInduced Suppression of LPS-Stimulated TNF- $\alpha$ Production from Human THP-1 Cells. Cells were preincubated with mifepristone alone (50 and $100 \mu \mathrm{M})$, mifepristone and idoBR1 $(10 \mu \mathrm{M})$, or mifepristone and $50 \mu \mathrm{M}$ DM for $24 \mathrm{~h}$ and then incubated for a further $20 \mathrm{~h}$ in the absence or presence of LPS $(10 \mu \mathrm{g} / \mathrm{mL})$. Following incubation at $37{ }^{\circ} \mathrm{C}\left(5 \% \mathrm{CO}_{2}\right.$, $100 \%$ humidity), the medium was collected from each culture well, and the levels of TNF- $\alpha$ were measured by ELISA. In order to preclude the possibility that the suppressive effect of idoBR1 may have been mediated via increased cell death, the effect of idoBR1 $(10 \mu \mathrm{M})$ was assessed on cell viability by two methods: (a) enumeration of nonviable cells by Trypan blue uptake and (b) by MTT dye conversion. Following incubation,
Trypan blue was added to cells immediately before counting (using a hemocytometer), and the percentage of blue cells was subtracted from the total cell count. For MTT dye conversion, following incubation, $50 \mu \mathrm{L}$ of the medium was removed from all wells and replaced with $50 \mu \mathrm{L}$ of $4 \mathrm{mg} / \mathrm{mL}$ MTT (Sigma) in sterile PBS. Cells were incubated at $37{ }^{\circ} \mathrm{C}$ for further $4 \mathrm{~h}$, and then, $100 \mu \mathrm{L}$ of DMSO was added to each well, and absorbances were measured at $570 \mathrm{~nm}$ (SpectraMax Microplate Reader, Molecular Devices).

Serum Cytokine Study in Healthy Humans Given Cucumber Extract. Serum cytokines were measured over 4 days in healthy human subjects (white caucasian male, mean weight $73 \mathrm{~kg}$, and ages 20-25) pre- and post-intense exercise [placebo $n=7$ and cucumber extract (Q-actin) treated $n=10$ ]. The subjects were given Q-actin $(20 \mathrm{mg})$ or placebo in capsules daily, including before the first exercise. Venous blood samples were collected prior to the exercise and on days 1, 2, 3, and 4 post-muscle damage using the standard phlebotomy technique from an antecubital vein. Blood was collected into a serum separator tube and allowed to clot. Once clotted, the blood was centrifuged ( $1500 \mathrm{~g}$ for $20 \mathrm{~min}$ ). The isolated serum was frozen at $-80{ }^{\circ} \mathrm{C}$ until cytokine analysis. Serum concentrations of TNF- $\alpha$, IL-10, and IL-12p70 were measured using a multiplex technique. ${ }^{14}$ Briefly, aliquots of the serum were analyzed in duplicates using a high-sensitivity bead-based multiplex assay, according to the manufacturer's recommendations (MILLIPLEX; EMD Millipore; St. Louis, MO).

Glycosidase Activity Assay. BR1 is reported to be an inhibitor of iduronidase. ${ }^{8}$ BR1 and idoBR1 also have some structural features suggesting that they may be analogues of sialic acids. We, therefore, assayed $\mathrm{BR} 1$, idoBR1, and the related $\mathrm{DNJ}$ in a panel of glycosidases to determine the selectivity of inhibition. For the $\alpha$-L-iduronidase assay, solutions of $0.2 \mathrm{M}$ sodium formate buffer at $\mathrm{pH} 3.5$ and 0.2 $\mathrm{mM}$ substrate 4-methylumbelliferyl- $\alpha$-L-iduronide (Glycosynth, 44076), dissolved in buffer, were prepared. A solution of recombinant human $\alpha$-L-iduronidase (IDUA, R\&D Systems, 4119-GH) was also prepared at between $0.8 \mu \mathrm{g} / \mathrm{mL}$ in the buffer. The incubation mixture consisted of $10 \mu \mathrm{L}$ of enzyme solution, $10 \mu \mathrm{L}$ of $1 \mathrm{mg} / \mathrm{mL}$ aqueous idoBR1 and BR1 solution, and $20 \mu \mathrm{L}$ of $0.2 \mathrm{mM}$ substrate made up in buffer at $\mathrm{pH}$ 3.5. The reactions were carried out at $27^{\circ} \mathrm{C}$ and stopped by removing a $10 \mu \mathrm{L}$ aliquot and adding it to $200 \mu \mathrm{L}$ of $0.5 \mathrm{M}$ glycine $(\mathrm{pH} 10.4)$ in a black-well plate during the exponential phase of the reaction, which had been determined at the beginning using uninhibited assays in which water was replaced by an inhibitor. Final fluorescence values were read at excitation and emission wavelengths of 365 and $445 \mathrm{~nm}$, respectively, using a SpectraMax microplate reader (Molecular Devices). Assays were carried out in triplicates. Results were expressed as a percentage of uninhibited assays in which water was replaced by an inhibitor.

Sialidase assays used $10 \mu \mathrm{L}$ of $1 \mathrm{mg} / \mathrm{mL}$ idoBR1 (or water without an inhibitor) and $10 \mu \mathrm{L}$ of $2.5 \mathrm{nM}$ sialidase (NanH from Tannerella forsythia- a broad specificity GH33 bacterial enzyme) incubated in the presence of $30 \mu \mathrm{L}$ of $0.1 \mathrm{mM}$

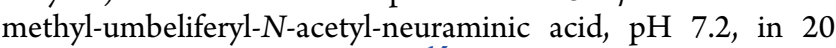
$\mathrm{mM}$ sodium phosphate buffer. ${ }^{15}$ Oseltamivir was used as a positive control. Reactions were stopped at 60, 120, and $180 \mathrm{~s}$ using $100 \mu \mathrm{L}$ of $100 \mathrm{mM}$ sodium carbonate buffer, $\mathrm{pH} 10.5$, added to $50 \mu \mathrm{L}$ of the reaction mixture. Release of methylumbelliferone was quantified by measuring fluorescence emission at $450 \mathrm{~nm}$ and excitation at $350 \mathrm{~nm}$. Reactions 
were carried out in triplicates. The percentage of sialidase activity was expressed as the change in fluorescence compared to that of a reaction without inhibitors.

Other glycosidase assays used p-nitrophenyl-glycopyranosides and were carried out using the iminosugar acids idoBR1 and BR1 as available, and the results are compared with the related iminosugar DNJ (3). All enzymes and p-nitrophenylsubstrates were purchased from Sigma, with the exception of $\beta$ mannosidase which was obtained from Megazyme. Enzyme reactions were carried out at $27^{\circ} \mathrm{C}$ in $0.1 \mathrm{M}$ citric acid/0.2 M disodium hydrogen phosphate buffers at the optimum $\mathrm{pH}$ for the enzyme. The incubation mixture consisted of $10 \mu \mathrm{L}$ of enzyme solution, $10 \mu \mathrm{L}$ of $1 \mathrm{mg} / \mathrm{mL}$ aqueous solution of the extract, and $50 \mu \mathrm{L}$ of the appropriate $5 \mathrm{mM} p$-nitrophenyl substrate made up in buffer at the optimum $\mathrm{pH}$ for the enzyme. The reactions were stopped by the addition of $70 \mu \mathrm{L}$ of $0.4 \mathrm{M}$ glycine ( $\mathrm{pH}$ 10.4) during the exponential phase of the reaction. This phase had been determined at the beginning using uninhibited assays in which water was replaced by an inhibitor. Final absorbances were measured at $405 \mathrm{~nm}$ using a VersaMax microplate reader (molecular devices). Assays were carried out in triplicates, and the values given are means of the three replicates per assay. Results were expressed as \% inhibition compared to the controls without inhibitors.

Cell Culture Sialidase Activity Assay. There can be differences in enzymes from different sources, and so we wanted to study human-derived as well as bacterial sialidases. As the human sialidase enzymes are not readily commercially available, we used activities from cell cultures.

THP-1 Cell Line Treatment for the Sialidase Activity Test. The THP-1 cells were cultured in Roswell Park Memorial Institute (RPMI) 1640 media supplemented with 10\% FCS, $2 \beta$-mercaptoethanol, and glutamine to get $80 \%$ confluence in culture flasks. The culture was aspirated and centrifuged at $1500 \mathrm{rpm}$ for $5 \mathrm{~min}$, and then, the pellet was resuspended in 1 $\mathrm{mL}$ of RPMI complete media and counted conventionally using a hemocytometer. The cells $\left(5 \times 10^{6}\right)$ were incubated with PMA $(10 \mathrm{ng} / \mathrm{mL})$ in separate dishes (stimulates the PKC pathway) to form differentiated adherent macrophages. To determine the sialidase activity, the adherent cells were pretreated for $1 \mathrm{~h}$ with idoBR1 and Q-actin extracts, at the concentrations 100 to 12.5 and 200 to $25 \mu \mathrm{g} / \mathrm{mL}$ respectively serial diluted twofold, and DM at 100 and $1000 \mathrm{ng} / \mathrm{mL}$ as a positive control followed by $24 \mathrm{~h}$ LPS $(1 \mu \mathrm{g} / \mathrm{mL})$ stimulation. Post incubation, the cells were carried over to determine the sialidase activity. The sialidase activity was found to be maximum at the $16 \mathrm{~h}$ incubation time point with LPS.

Determination of Endogenous Sialidase Activity. THP-1 cells were washed with phosphate-buffered saline and resuspended in ice-cold buffer containing $0.25 \mathrm{M}$ sucrose, 1 mM EDTA, and $0.2 \mathrm{mM}$ phenylmethylsulfonyl fluoride. The cell suspension was sonicated on ice for $15 \mathrm{~s}$ on a low setting (6\% amplitude) (VibraCell; Sonics and Materials Inc., Newtown, CT) followed by centrifugation at $25,000 \mathrm{~g}$ for 15 min at $4{ }^{\circ} \mathrm{C}$. The resultant supernatant was used to determine the lysosomal sialidase activity. Protein quantification of the supernatant was performed using the Bio-Rad protein determination kit as described above. For the determination of lysosomal sialidase activity, $200 \mu \mathrm{g}$ of the total protein was mixed with 40 nmol 4-methyl-umbelliferyl- $\alpha$ - $N$-acetyl-Dneuraminic acid (Sigma), the lysosomal sialidase-specific substrate, $10 \mu \mathrm{mol}$ sodium acetate buffer, $\mathrm{pH} 4.6$, and 200 $\mu \mathrm{g}$ of bovine serum albumin in a total volume of $200 \mu \mathrm{L}$. The sialidase reaction was allowed to proceed for $1 \mathrm{~h}$ at $37^{\circ} \mathrm{C}$ and was terminated by the addition of $0.25 \mathrm{M}$ glycine $\mathrm{NaOH}, \mathrm{pH}$ 10.4. The released 4-methyl-umbelliferyl- $N$-acetyl-D-neuraminic acid was measured as fluorescence (Synergy 2 multimode microplate reader) at an excitation wavelength of $365 \mathrm{~nm}$ and an emission wavelength of $448 \mathrm{~nm}$.

ELISA of Cytokines Produced by THP-1 Monocytic Cell-Derived Macrophages. Sample Preparation for ELISA. The cells were aspirated from an $80 \%$ confluence culture flask and centrifuged at $1500 \mathrm{rpm}$ for $5 \mathrm{~min}$. The cell pellet was then resuspended in $1 \mathrm{~mL}$ of RPMI complete media, and $1 \times 10^{5}$ cells were seeded to each well of a 96-well microtiter plate. After $24 \mathrm{~h}$ incubation, PMA $(10 \mathrm{ng} / \mathrm{mL})$ was added to the 96 well plate to differentiate the THP-1 cells. To determine the range of cytokine production, the differentiated adherent THP1 cells (macrophages) were pretreated for $1 \mathrm{~h}$ with idoBR 1 and Q-actin extract at the concentrations 200 to $25 \mu \mathrm{g} / \mathrm{mL}$ diluted twofold serially, followed by $2 \mathrm{~h}$ LPS $(100 \mathrm{ng} / \mathrm{mL})$ stimulation, as reported by others using similarly differentiated THP-1 cells $^{16}$ and also because trials showed measurable cytokines and we were mainly interested in the signaling pathways here. Post incubation, the cell supernatant from each of the wells was aspirated into sterile microcentrifuge tubes and centrifuged at $1000 \mathrm{rpm}$ for 2-3 min to settle any cells present. The cell supernatant was then used for evaluation of the presence of TNF- $\alpha$ and other selected cytokines using ELISA.

Sandwich ELISA. ELISA plates coated with antibodies for TNF- $\alpha$, IL-12, IL-1 $\beta$, IL- $1 \alpha$, or IL-10 (R\&D Systems, USA) were used following the manufacturer's instructions. Optical density (OD) was measured within $30 \mathrm{~min}$ after adding the stop solution at $450 \mathrm{~nm}$.

Determination of CD44-Bound HA Using Modified ELISA. The anti-CD44 monoclonal antibody (Invitrogen, $2 \mu \mathrm{g}$ ) was coated to each well in 96-well plates in $50 \mathrm{mM}$ carbonate/ bicarbonate buffer ( $\mathrm{pH} 9.6)$ and incubated overnight at $4{ }^{\circ} \mathrm{C}$. The unbound antibodies were washed out using PBS with $0.05 \%$ Tween 20 (PBST wash solution). The wells were blocked using $1 \%$ BSA and incubated for $1 \mathrm{~h}$ at $37^{\circ} \mathrm{C}$. The wells were washed thoroughly three times using PBST wash solution by adding $200 \mu \mathrm{L}$ into the wells. Cell lysates $(50 \mu \mathrm{L})$ were added to the wells, and plates were incubated for $1 \mathrm{~h}$ at $37^{\circ} \mathrm{C}$. The wells were then washed three times by adding 200 $\mu \mathrm{L}$ of PBST and soaking the wells for $30 \mathrm{~s}$. The biotinylatedhyaluronic acid (HA) antibody followed by streptavidin-HRP was added to form an immune complex, and after incubating for $60 \mathrm{~min}$ at $37^{\circ} \mathrm{C}$, the solution was aspirated, and the wells were washed thrice with $200 \mu \mathrm{L}$ of the wash solution by soaking the wells for $30 \mathrm{~s}$. Both chromogen A and chromogen $\mathrm{B}(50 \mu \mathrm{L}$ each $)$ were added to each well. The plate was incubated for $15 \mathrm{~min}$ in the dark at $37{ }^{\circ} \mathrm{C}$. The reaction was stopped by adding $50 \mu \mathrm{L}$ of the stop solution, and absorbance was read at $450 \mathrm{~nm}$.

MAPK Signaling Effects of idoBR1 and Cucumber Extract Containing idoBR1. THP-1 cells were cultured in RPMI media supplemented with mercaptoethanol and glutamine to get $80 \%$ confluence in culture flasks; they were then aspirated and centrifuged at $1500 \mathrm{rpm}$ for $5 \mathrm{~min}$. The cell pellet was resuspended in $1 \mathrm{~mL}$ of RPMI complete media and counted conventionally using a hemocytometer. The cells $(5 \times$ $\left.10^{6}\right)$ were incubated with PMA $(10 \mathrm{ng} / \mathrm{mL})$ in separate dishes to differentiate THP-1 cells into adherent macrophages. To determine the protein expression p38 and p42/44, the adherent cells were pretreated for $1 \mathrm{~h}$ with idoBR $1-100$ 


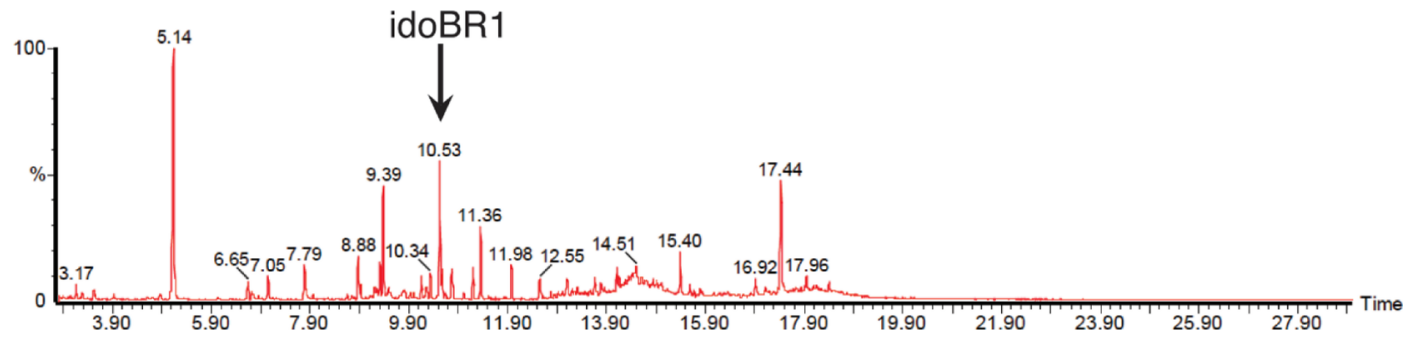

Figure 2. GCMS chromatogram of cucumber extract (TMS) showing idoBR1 at $10.53 \mathrm{~min}$.
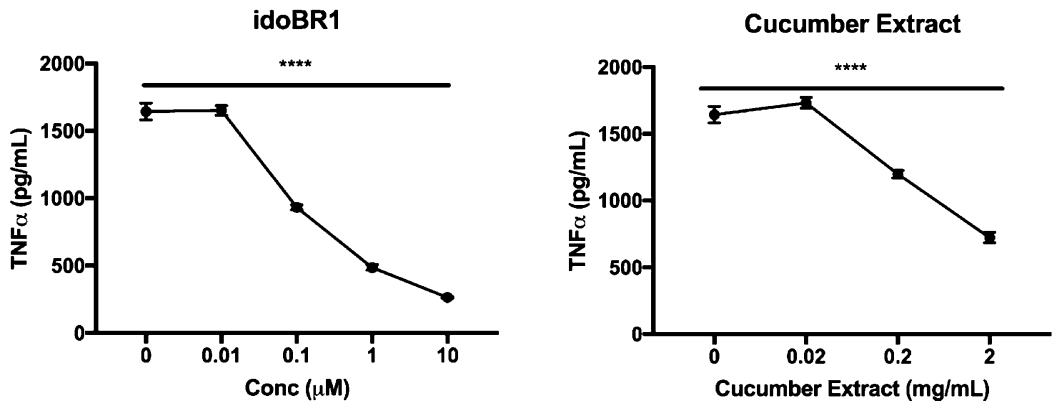

Figure 3. idoBR1 reduces TNF- $\alpha$ levels in LPS-stimulated human plasma.

and $50 \mu \mathrm{g} / \mathrm{mL}$ - and Q-actin extract-200 and $100 \mu \mathrm{g} / \mathrm{mL}$ followed by $2 \mathrm{~h}$ LPS $(1 \mu \mathrm{g} / \mathrm{mL})$ stimulation. DM was used as a positive control. Post incubation, the cells were harvested, and the whole protein was isolated.

Western Blot Procedure. Cell pellets were lysed, and the protein concentration was determined using the Bio-Rad protein determination assay (Bio-Rad). Total cell proteins were subjected to $8 \%$ polyacrylamide SDS gel electrophoresis followed by transferring onto polyvinylidene difluoride membranes (Thermo Scientific). The membranes were probed with either mouse anti-phospho-p38 mAb (Thermo Scientific) or mouse anti-phospho-p42/44 mAb (Thermo Scientific), followed by horseradish peroxidase-conjugated goat antimouse polyclonal antibodies (Thermo Scientific). All immunoblots were visualized by ECLTM Western Blotting Reagent (Amersham Biosciences).

Oral Consumption and Excretion of idoBR1. Q-actin $(1.0 \mathrm{~g})$ was dissolved in water and drunk with sliced tomato at mid-day by one adult white male (60, BMI 19) and one female (52, BMI 17.5). The healthy volunteers ate no other Cucurbits for $15 \mathrm{~h}$ prior to the experiment. Urine samples before consumption were collected over $3 \mathrm{~h}$ as $t=0$, and then, samples were collected for $9 \mathrm{~h}$ for the female and $15 \mathrm{~h}$ for the male. Q-actin $(0.5 \mathrm{~g})$ was extracted in water for $15 \mathrm{~h}, 100 \mu \mathrm{L}$ of $1 \mathrm{mg} / \mathrm{mL}$ castanospermine was added as an internal reference, and the idoBR 1 content was calculated as $1.4 \%$ by GCMS after cation exchange (Amberlite IR120 $\mathrm{H}^{+}$) chromatography. Each person, therefore, consumed $14 \mathrm{mg}$ of idoBR1. The entire urine samples were similarly treated using an excess of cation exchange resin, except the material displaced by ammonia solution was run a second time through the same cation exchange resin (now in the ammonium form) to reduce strong bases (which bind to IR120 resin in the ammonium form), and only the unretained material was kept. The urine idoBR1 fractions were dried and made up to $20 \mathrm{~mL}$ in water. Then 500 $\mu \mathrm{L}$ of each was sampled, and $25 \mu \mathrm{g}$ of castanospermine added.

\section{RESULTS AND DISCUSSION}

To identify specific compounds in cucumber that could be used as dietary supplements to reduce inflammation, we began by chemically fractionating the extract from the cucumber $C$. sativus. We identified the iminosugar acid idoBR1 (1) in the extract by comparison with an authentic synthetic material. ${ }^{11}$ As described here, the purified compound and extract containing it showed an inhibition of TNF- $\alpha$ production in human blood and cultured THP-1 cells. idoBR1 is the C5 epimer of the iminosugar acid BR1 (2), related to the iminosugar DNJ (3). DNJ is perhaps the most commonly occurring natural iminosugar and a potent inhibitor of glucosidases; it is currently used as the basis of a therapeutic agent in Miglitol and Miglustat for diabetes and type I Gaucher's disease, respectively. ${ }^{17}$ BR1 is the corresponding amino acid of DNJ; BR1, unlike DNJ, is not a strong inhibitor of any glycosidase but has, nonetheless, been shown to have potential for use in control of diabetes. ${ }^{18}$ GCMS analysis of cucumber extract as a TMS mixture shows idoBR1 as the component at $10.53 \mathrm{~min}$ retention time (Figure 2); it has a distinctive mass spectrum with major fragments at 217 (100\%), 258 (50\%), 348 (25\%), 375 (10\%), and 450 (5\%) amu. BR1 gives a similar mass spectrum with a retention time of 11.13 min under the same conditions. A second pertrimethylsilyl derivative of idoBR1 with a major fragment at $420 \mathrm{amu}$ is always seen at $20 \mathrm{~s}$ longer retention time but is never observed with BR1. Other peaks in the spectra are other molecules in the cucumber extract including sugars and amino acids. Cucumber does not always contain idoBR1 and is unusual among iminosugar-producing plants $^{19}$ that it only accumulates the single iminosugar amino acid and no iminosugars. The Q-actin extract used in the biological studies described here contained $1.4 \%$ idoBR $1(\mathrm{w} / \mathrm{w})$ as determined with an internal reference (castanospermine, retention time $11.98 \mathrm{~min}$ ). Although not found to be straight forward for quantification in extracts, LCMS using a triple quadrupole mass spectrometer and electrospray ionization (ESI) (Thermo 
Scientific) gave $178.01 \mathrm{~m} / z$ positive mode and $176.01 \mathrm{~m} / \mathrm{z}$ negative mode for idoBR1.

Evaluation of the Anti-Inflammatory Effects of idoBR1 and Q-Actin in Human Blood. TNF- $\alpha$, one of the most important proinflammatory cytokines, is secreted mainly from activated monocytes/macrophages in response to diverse extracellular stimuli and plays a major role in acute and chronic inflammatory diseases including septic shock and rheumatoid arthritis, ${ }^{20}$ respectively. We tested whether treatment of human blood with idoBR1 and Q-actin could reduce the production of the inflammatory cytokine TNF- $\alpha$ upon LPS stimulation. Indeed, when blood was incubated with compounds or extracts for $48 \mathrm{~h}$ prior to LPS stimulation, there were dose-dependent significant reductions in TNF- $\alpha$ release (Figure 3). These data suggest that idoBR1 and Q-actin may dampen the inflammatory response of blood/circulating immune cells.

The inhibition of LPS-induced TNF- $\alpha$ production in ex vivo human blood was strong ( $50 \%$ inhibition at $0.1 \mu \mathrm{M}$ for idoBR1 in blood) when there was preincubation with the compound or extract $(50 \%$ reduction at ca. $1 \mathrm{mg} / \mathrm{mL}$. of extract with $>1 \%$ idoBR1 (equating to $>56 \mu \mathrm{M}$ idoBR1), confirming that idoBR1 alone can be responsible for the anti-inflammatory effect of cucumber extract). Preincubation of human plasma with idoBR1 and Q-actin cucumber extract prior to LPS stimulation for $20 \mathrm{~h}$ significantly reduced TNF- $\alpha$ levels. Statistical significance was determined with one-way ANOVA.

Under similar incubation conditions used for the human plasma study but using human THP-1 cells, the inhibitory action of idoBR1 (at $10 \mu \mathrm{M}$ ) was comparable to that of identical pretreatment with DM $(50 \mu \mathrm{M})$ with an inhibition of $>50$ and $>65 \%$, respectively. Mifepristone alone with LPS greatly amplified TNF- $\alpha$ production from the THP-1 cells; however, in the presence of DM, it reversed the suppressive action of DM but not that of idoBR1. As mifepristone is primarily a progesterone receptor antagonist, this indicates that idoBR1 is unlikely to act via a glucocorticoid or progesterone receptor. idoBR1 $(10 \mu \mathrm{M})$ in the absence or presence of LPS $(10 \mu \mathrm{g} / \mathrm{mL})$ did not reduce the viability of the THP-1 cells and indeed may have had a small cytoprotective action, especially in the presence of LPS.

Serum Cytokine Study in Healthy Humans Given Cucumber Extract. The 4 day pilot study involving intense periods of resistance exercise with consumption of cucumber extract with $20 \mathrm{mg}$ of $1.4 \%$ idoBR1 (iminosugar) or placebo 1 $\mathrm{h}$ prior to exercise showed a trend for cucumber extract controlling inflammatory cytokines with TNF- $\alpha$ appearing to be held fairly constant (Figure 4a), while IL-10 (Figure 4b) and the Th-1 cytokine IL-12p70 (Figure 4c) showing a trend to be increased on cucumber extract consumption combined with intense exercise. Interestingly, IL-12p70 is considered proinflammatory but has an important role in preventing infections, for example, viruses. ${ }^{21,22}$

idoBR1 Reduces Sialidase Activity. DNJ is a potent inhibitor of $\alpha$ - and $\beta$-glucosidases. ${ }^{19}$ To determine whether idoBR1 was also a glycosidase inhibitor, assays were performed to measure the effects of idoBR1 on a panel of glycosidases. idoBR1 most potently, selectively, inhibited broad-specificity bacterial sialidase (Table 1 ) with $60 \%$ inhibition at $0.8 \mathrm{mM}$ $\left(\mathrm{IC}_{50} 0.5 \mathrm{mM}\right.$; oseltamivir had an $\mathrm{IC}_{50}$ of $0.25 \mathrm{mM}$ in the same assay). Bacterial sialidase is a reported virulence factor that contributes to inflammatory responses by modifying the glycosylation status of toll-like receptors (as well as other
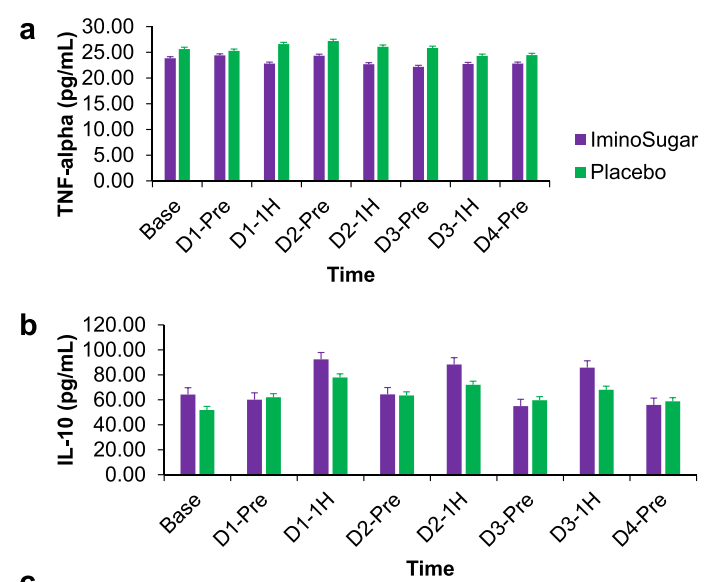

C

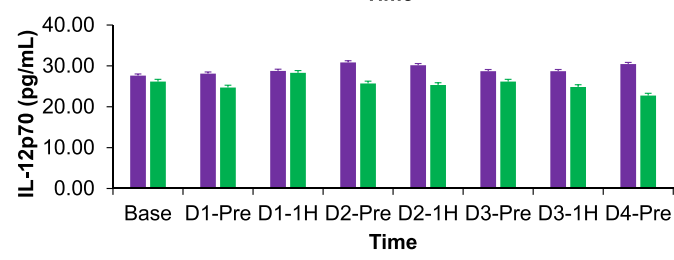

Figure 4. (a) Mean serum before and after intense exercise TNF- $\alpha$ was not increased in the subjects given cucumber extract (iminosugar) $1 \mathrm{~h}$ before exercise compared to the placebo group. (b) Mean before and after intense exercise IL-10 serum levels in the cucumber extract subjects (purple) compared to the placebo group (green). (c) Mean before and after intense exercise IL-12p70 levels in the serum of those given cucumber extract (purple) compared to those given the placebo (green).

receptors involved in inflammation such as integrins and EGFR). ${ }^{23}$ DNJ was not inhibitory to the same sialidase at 0.8 $\mathrm{mM}$. idoBR1 was not strongly inhibitory to any of the other glycosidases assayed, but it was weakly inhibitory to $\alpha$-Liduronidase with an $\mathrm{IC}_{50}$ of approximately the concentration tested $(1.5 \mathrm{mM})$. As expected from Manning et al., ${ }^{8}$ BR1 here gave moderate inhibition of $\beta$-glucuronidase at the concentration used; in contrast, DNJ was a potent inhibitor of Bacillus but not yeast $\alpha$-glucosidases and also showed significant inhibition of $\beta$-glucosidase. These data show that idoBR1 and BR1 are moderate glycosidase inhibitors but are selective in their inhibition profiles. A further study looking at inhibition of human sialidase activity induced in THP-1 cells treated with LPS also showed an inhibitory effect (Figure 5). The standard idoBRl tested at 0.28 and $0.56 \mathrm{mM}$ showed a maximum reduction in relative sialidase activity of 0.63 and 0.55 , respectively, compared to the LPS control. Q-actin at 100 and $200 \mu \mathrm{g} / \mathrm{mL}$ showed a maximum reduction of relative sialidase activity to 0.7 and 0.62 , respectively, compared to the control (LPS). The result of this study could be a combination of reduced expression of sialidase or inhibition of the enzyme by idoBR1.

Interestingly, an induced sialidase is proposed to be involved in inflammatory responses mediated by CD $44 .{ }^{24}$ CD 44 has been shown to be involved in hemopoiesis, homing to the mucosal lymphatic tissue, and lymphocyte infiltration into inflammatory tissues. ${ }^{25}$ HA interactions with CD44 and CD168 (RHAMM) can induce numerous cell behaviors, including activation of tyrosine kinases, protein kinase $\mathrm{C}, \mathrm{FAK}$, $\mathrm{Pi} 3 \mathrm{~K}$, mitogen-activated protein kinases (MAPK), $\mathrm{NF} \kappa \mathrm{B}$, and RAS, as well as cytoskeletal components required for inflammation and cancer. Although most cells express some 
Table 1. Comparison of the Glycosidase Inhibitions (\% Inhibition) by idoBR1, BR1, and DNJ ${ }^{a}$

\begin{tabular}{lllll}
\multicolumn{1}{c}{ enzyme } & \multicolumn{1}{c}{ source } & \% inhib. by idoBR1 (1) & \% inhib. by BR1 (2) & \% inhib. by DNJ (3) \\
$\alpha$-D-glucosidase & Saccharomyces cerevisiae & 0 & 0 & 36 \\
$\alpha$-D-glucosidase & Bacillus sterothermophilus & 26 & 49 & $100(\mathrm{IC} 50$ 2.6 $\mu \mathrm{M})$ \\
$\beta$-D-glucosidase & almond & 0 & 7 & 64 \\
$\alpha$-D-galactosidase & green coffee bean & 0 & 0 & 0 \\
$\beta$-D-galactosidase & bovine liver & 0 & 0 & 0 \\
$\alpha$-D-mannosidase & jack bean & -28 & 29 & 27 \\
$\beta$-D-mannosidase & Cellullomonas fimi & 0 & 8 & -19 \\
$N$-acetyl- $\beta$-D-glucosaminidase & bovine kidney & 0 & 0 & 0 \\
$N$-acetyl- $\beta$-D-glucosaminidase & jack bean & 0 & 10 & 0 \\
$\beta$-D-glucuronidase & bovine liver & 0 & 89 & ND \\
$\alpha$-L-iduronidase & recombinant human & 48 & 9 & ND \\
sialidase & T. forsythia & $60\left(\mathrm{IC}_{50} 0.5 \mathrm{mM}\right)$ & $\mathrm{ND}$ & $\mathrm{ND}$
\end{tabular}

${ }^{a} \mathrm{ND}=$ not determined. All compounds were assayed at $0.8 \mathrm{mM}$ (except for $1.4 \mathrm{mM}$ for iduronidase). Negative values show promotion of the enzyme activity.

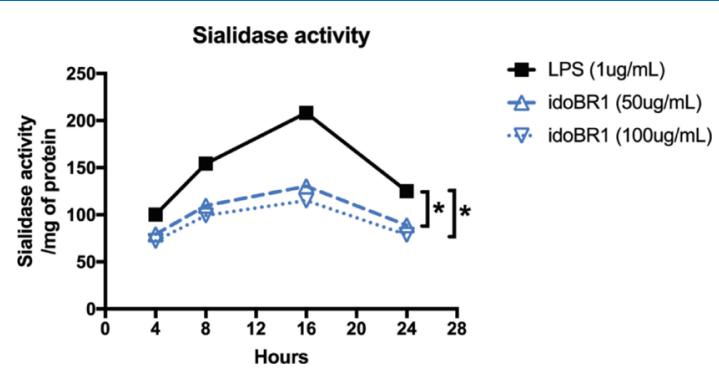

Figure 5. Sialidase inhibition by idoBR1 following LPS stimulation in THP-1 cells. Sialidase activity was significantly reduced in cells treated with idoBR1 in the presence of LPS at both concentrations. Statistical significance determined by two-way ANOVA.

form of CD44, not all cells constitutively bind to HA. ${ }^{26,27}$ Functionally active HA-adhesive CD44 is produced through induction of sialidase via MAPK activation.

Studies carried out to understand the role of MAPK in the LPS-induced inflammatory response show MAPK p42/44mediated TNF- $\alpha$ production and subsequent TNF- $\alpha$-mediated p38 activation, resulting in the production of HA-adhesive CD44 by sialidase activity. ${ }^{28}$ Thus, a possible mechanism for the anti-inflammatory activity of idoBR 1 is via inhibition of an induced sialidase, and this sialidase may interact directly with inflammatory receptors such as TLRs. ${ }^{29}$

Investigations of Signaling Pathways Involving idoBR1 and Q-Actin and Associated Levels of Inflammatory Markers in Cell-Based Assays. Next, we tested whether isolated idoBR1 and the standardized cucumber extract $\mathrm{Q}$-actin, that contains $>1 \%$ idoBR1, could reduce the inflammatory response of LPS-stimulated human monocytes (differentiated adherent macrophages and THP-1 cell-derived) and studied signaling. Even with a fairly short incubation time with LPS, the amount of released TNF- $\alpha$ was significantly reduced from low to high concentrations of both idoBR1 and Q-actin (Figure 6A). Concurrent with a reduction in TNF- $\alpha$ levels is the reduction of a downstream signaling product of TNF- $\alpha$, the association of HA with the CD44 receptor (Figure $6 \mathrm{~F}$ ). The CD44 binding study which showed CD44-bound HA was $110 \mathrm{ng} / \mathrm{mL}$ in the LPS-stimulated $(1 \mu \mathrm{g} / \mathrm{mL})$ THP- 1 cellderived macrophages, whereas ido-BR1 at $100 \mu \mathrm{g} / \mathrm{mL}$ gave a reduction of CD44-HA levels (27\%) as compared to the LPS control. Cucumber extract Q-actin at $200 \mu \mathrm{g} / \mathrm{mL}$ also gave a reduction of CD44-HA levels (31\%) in THP-1 cells as compared to the LPS control. DM, the positive control, gave a reduction of $24 \%$ at $100 \mathrm{ng} / \mathrm{mL}$ and $42 \%$ at $1000 \mathrm{ng} / \mathrm{mL}$.

TNF- $\alpha$ can also synergize with other cytokines, notably interleukin-1 (IL-1), to aggravate many inflammatory responses. $^{30,31}$ Therefore, we also analyzed the release of IL$1 \alpha$ and IL- $1 \beta$ and found that idoBR 1 significantly reduced levels of both IL- $1 \alpha$ and IL- $1 \beta$ from 25 to $100 \mu \mathrm{g} / \mathrm{mL}$ and Qactin from 50 to $100 \mu \mathrm{g} / \mathrm{mL}$ (Figure 6C,D). Similarly, a significant reduction was observed for IL-12 release (note not so in the humans given intense exercise over a longer period), which acts to stimulate IFN- $\gamma$ and TNF- $\alpha$ production in $\mathrm{T}$ lymphocytes (Figure 6B). In addition to a reduction in the production of proinflammatory cytokines, we also observed a significant increase in the release of the anti-inflammatory cytokine IL-10 at all concentrations of idoBR 1 and from 25 to $100 \mu \mathrm{g} / \mathrm{mL}$ Q-actin (Figure 6E). These data suggest that idoBR1 and Q-actin potentially reduce inflammatory responses in LPS-stimulated human monocytes, perhaps by reducing the activity of an induced sialidase involved in CD44-HA binding.

MAPK Signaling Effects of idoBR1 and Cucumber Extract Containing idoBR1. MAPK signaling cascade plays an essential role in the initiation of inflammatory responses. The induction of inflammatory cytokine genes requires activation of the MAPKs and stimulation of extracellular regulated protein kinase/MAPK (ERK/MAPK) pathways essential for downstream inflammatory responses. ${ }^{32,33}$ The MAPK pathway is also required for the expression of inflammatory mediator genes, including COX-2, iNOS, IL$1 \beta$, and TNF- $\alpha$. It was reported that the ERK and/or p38 MAPK are involved in upregulation of IL- $1 \beta \cdot{ }^{34}$ Q-actin tested at 100 and $200 \mu \mathrm{g} / \mathrm{mL}$ showed relative reduction in phosphorylated p38 expression of 0.92 and 0.83 , respectively, compared to the LPS control. In the case of idoBR1 at 50 and $100 \mu \mathrm{g} / \mathrm{mL}$, it showed reductions in phosphorylated p38 expression of 0.88 and 0.80 , respectively, compared to the LPS control. Q-actin tested at 100 and $200 \mu \mathrm{g} / \mathrm{mL}$ showed relative reductions in phosphorylated ERK $42 / 44$ expression of 0.81 and 0.78 , respectively, compared to the LPS control (Tables 2 and 3). idoBR1 at 50 and $100 \mu \mathrm{g} / \mathrm{mL}$ showed reductions in phosphorylated ERK 42/44 expression of 0.80 and 0.76 , respectively, compared to the LPS control. Both idoBR1 and cucumber extract containing idoBR1 (Q-actin) are, therefore, shown to be able to reduce the MAPK signaling cascade that plays an essential role in the inflammatory response. 


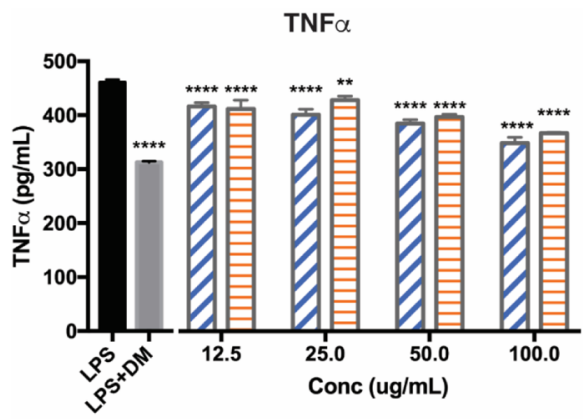

A

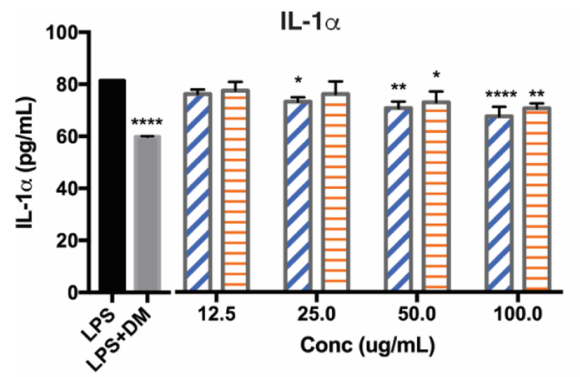

C

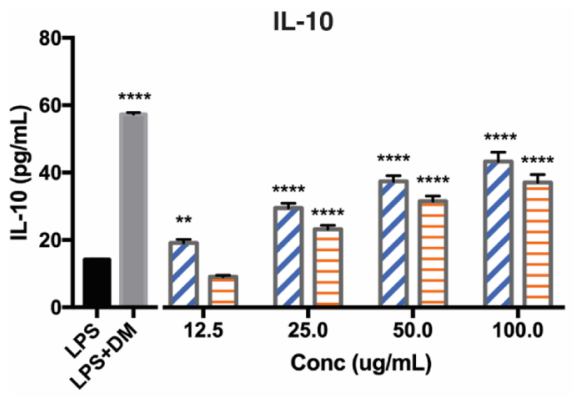

E

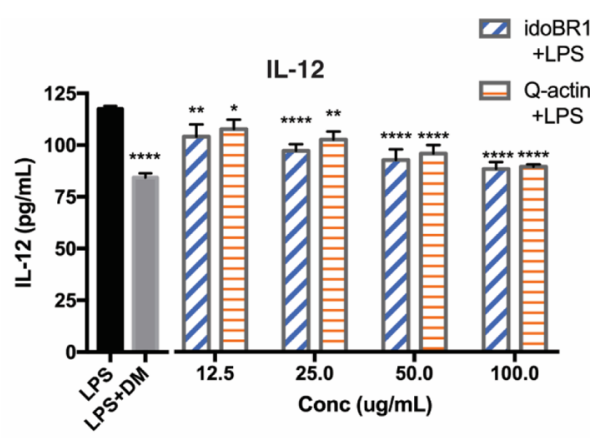

B

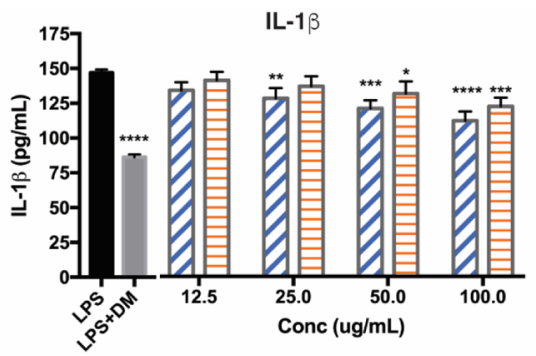

D

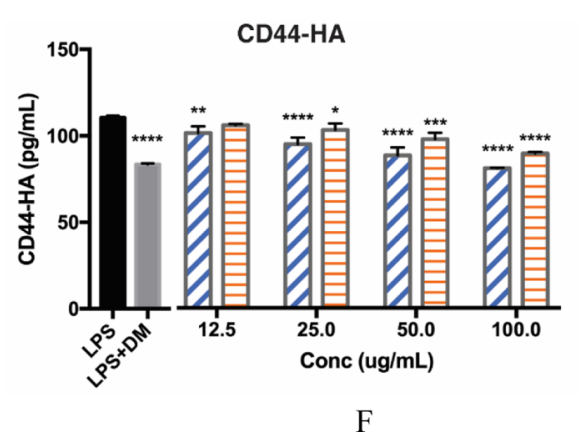

Figure 6. idoBR1 reduces LPS-induced increase in proinflammatory cytokine release in human monocytes. THP-1 cells were stimulated with LPS in conjunction with the anti-inflammatory steroid analogue, DM, idoBR1, or Q-actin and assessed for cytokine release following $2 \mathrm{~h}$ incubation. $(\mathrm{A}, \mathrm{B})$ TNF- $\alpha$ and IL-12 levels were elevated in the presence of LPS and significantly reduced by DM and at all concentrations of idoBR1 and Qactin. (C,D) IL- $1 \alpha$ and IL-1 $\beta$ levels were elevated by LPS and significantly reduced by DM. idoBR1 treatment shows significant reduction at $25-$ $100 \mu \mathrm{g} / \mathrm{mL}$, and Q-actin significantly reduced levels at $50-100 \mu \mathrm{g} / \mathrm{mL}$ concentrations; (E) anti-inflammatory IL-10 was significantly increased in $\mathrm{DM}$ and all concentrations of idoBR1 and Q-actin; (F) CD44-HA binding activity was significantly reduced in DM, all idoBR1, and at 25-100 $\mu \mathrm{g} /$ $\mathrm{mL} \mathrm{Q}$-actin concentrations. Statistical significance was determined with one-way ANOVA.

Table 2. Relative Expression of p42/44

$\begin{array}{lrc}\text { test samples } & \text { conc. }(\mu \mathrm{g} / \mathrm{mL}) & \text { relative expression } \\ \text { LPS } & 1 & 1.00 \\ \text { Q-actin } & 100 & 0.81 \\ \text { Q-actin } & 200 & 0.78 \\ \text { idoBR1 } & 50 & 0.80 \\ \text { idoBR1 } & 100 & 0.76 \\ \text { DM } & 1 & 0.65\end{array}$

The results show that idoBR1 gives inhibition of sialidases from both bacteria and human THP-1 cell cultures. Q-actin cucumber extract with $>1 \%$ idoBR1 was also shown to inhibit the human cell sialidase activity.

Oral Fate of idoBR1 in Humans. Finally, we conducted a pilot experiment to determine if by oral route idoBR1 was excreted largely unmetabolized in urine as other iminosugars studied previously in rats. ${ }^{35-37}$ Two adults consumed $1 \mathrm{~g}$ of $\mathrm{Q}$ actin in water, calculated to contain $14 \mathrm{mg}$ of idoBR1. idoBR1
Table 3. Relative Expression of p38

\begin{tabular}{lrc} 
test samples & conc. $(\mu \mathrm{g} / \mathrm{mL})$ & relative expression \\
LPS & 1 & 1.00 \\
Q-actin & 100 & 0.92 \\
Q-actin & 200 & 0.83 \\
idoBR1 & 50 & 0.88 \\
idoBR1 & 100 & 0.80 \\
DM & 1 & 0.54 \\
\hline
\end{tabular}

was clearly seen by GCMS in the urine. After $15 \mathrm{~h}$, the male showed excretion of approximately $6 \mathrm{mg}$ of idoBR 1 compared to the castanospermine reference peak area. The female had excreted approximately $9 \mathrm{mg}$ of idoBR 1 in $9 \mathrm{~h}$. It is reported in rats that less than $50 \%$ DNJ, fagomine, and 1,4-dideoxy-1,4imino-D-arabinitol ${ }^{37,38}$ are excreted in urine, although a more accurate ${ }^{15} \mathrm{~N}$-labeled $\mathrm{DNJ}$ study reports $80 \%$ excretion by rats over $48 \mathrm{~h},{ }^{24}$ and the authors conclude it may take longer than $48 \mathrm{~h}$ to be completely eliminated. Our method using cation 
exchange chromatography gave a useful concentration step that allowed clear identification of idoBR1 in human urine that would allow a more detailed availability and elimination study. It does appear though that idoBR1 is orally stable in humans and is largely excreted in urine over several hours. Anti-TNF therapies such as etanercept and infliximab show great promise against even intractable diseases such as Crohn's disease, but immune response to these proteins severely limits their prolonged use; ${ }^{39}$ idoBR1 or Q-actin may therefore have some advantages over these existing treatments.

In conclusion, idoBR1 in certain cucumbers has an antiinflammatory activity that may be due to sialidase inhibition or interaction with CD44-HA signaling. Regular consumption of cucumbers with idoBR1 may have a significant effect, as suggested by the effect of preincubation on TNF- $\alpha$ production in human plasma. Long-term use of steroids is not recommended, ${ }^{40}$ and commonly used nonsteroidal antiinflammatory drugs can also have several negative effects, including effects on the central nervous system. ${ }^{41}$ idoBR 1 is a nonsteroid and appears to have a novel mechanism. It appears nontoxic to humans, although its health significance in the diet should be studied further.

\section{ASSOCIATED CONTENT}

\section{(s) Supporting Information}

The Supporting Information is available free of charge at https://pubs.acs.org/doi/10.1021/acsomega.0c02092.

Additional GCMS data for the iminosugar amino acids idoBR1 and BR1 and the iminosugar DNJ; mass spectra of idoBR1; GCMS chromatogram of cucumber extract showing idoBR1; mass spectra of the two derivatives of idoBR1 in Q-actin; and chromatograms of human urine samples clearly showing the presence of idoBR1 following oral consumption of Q-actin cucumber extract (PDF)

\section{AUTHOR INFORMATION}

\section{Corresponding Author}

Robert J. Nash - PhytoQuest Limited, Ceredigion SY23 3EB, U.K.; (1) orcid.org/0000-0002-3272-640X; Phone: +44 1970 823200; Email: robert.nash@phytoquest.co.uk; Fax: +44 1970823209

\section{Authors}

Barbara Bartholomew - PhytoQuest Limited, Ceredigion SY23 3EB, U.K.

Yana B. Penkova - PhytoQuest Limited, Ceredigion SY23 3EB, U.K.

Dino Rotondo - Strathclyde Institute of Pharmacy and Biomedical Sciences, University of Strathclyde, Glasgow G4 ORE, U.K.

Fernanda Yamasaka - Strathclyde Institute of Pharmacy and Biomedical Sciences, University of Strathclyde, Glasgow G4 ORE, U.K.

Graham P. Stafford - Integrated BioSciences, School of Clinical Dentistry, University of Sheffield, Sheffield S10 2TA, U.K.

Sarah F. Jenkinson - Chemistry Research Laboratory, University of Oxford, Oxford OX1 3TA, U.K.

George W. J. Fleet - Chemistry Research Laboratory, University of Oxford, Oxford OX1 3TA, U.K.

Complete contact information is available at: https://pubs.acs.org/10.1021/acsomega.0c02092

\section{Notes}

The authors declare no competing financial interest.

\section{ACKNOWLEDGMENTS}

We acknowledge the excellent work of Dr. Brian McFarlin, University of North Texas for the exercise study.

\section{ABBREVIATIONS}

BR1, (2S,3R,4R,5S)-trihydroxypiperidine-2-carboxylic acid; idoBR1, $(2 R, 3 R, 4 R, 5 S)$-trihydroxypiperidine-2-carboxylic acid; DNJ, 1-deoxynojirimycin; TNF- $\alpha$, tumor necrosis factor alpha; OD, optical density; LPS, lipopolysaccharide; IL, interleukin; THP-1, a monocytic leukemia cell line; MAPK, mitogen-activated protein kinase; p38, p42, p44; classes of mitogen-activated protein kinases; $\mathrm{HA}$, hyaluronic acid; $\mathrm{NF} \kappa \mathrm{B}$, nuclear factor kappa-light-chain-enhancer of activated B cells; DM, dexamethasone; Pi3K, phosphoinositide 3-kinase; FAK, focal adhesion kinase; RAS, guanosine-nucleoside binding protein; ERK, extracellular signal-regulated kinase; amu, atomic mass unit; GCMS, gas chromatography-mass spectrometry

\section{REFERENCES}

(1) Renner, S. S.; Schaefer, H.; Kocyan, A. Phylogenetics of Cucumis (Cucurbitaceae): cucumber (C. sativus) belongs in an Asian/Australian clade far from melon (C. melo). BMC Evol. Biol. 2007, 7, 58.

(2) Pliny the Elder. Natural History; A.D. 79, reproduced by Bostock, J. and Riley, H. T. Taylor and Francis: London, 1855, Vol. 19, p 23.

(3) Mukherjee, P. K.; Nema, N. K.; Maity, N.; Sarkar, B. K. Phytochemical and therapeutic potential of cucumber. Fitoterapia 2013, 84, 227-236.

(4) Bernardini, C.; Zannoni, A.; Bertocchi, M.; Tubon, I.; Fernandez, M.; Forni, M. Water/ethanol extract of Cucumis sativus L. fruit attenuates lipopolysaccharide-induced inflammatory response in endothelial cells. BMC Compl. Alternative Med. 2018, 18, 194.

(5) Agatemor, U.; Nwodo, O.; Anosike, C. Anti-inflammatory activity of Cucumis sativus L. Br. J. Pharmaceut. Res. 2015, 8, 1-8.

(6) Trejo-Moreno, C.; Méndez-Martínez, M.; Zamilpa, A.; JiménezFerrer, E.; Perez-Garcia, M.; Medina-Campos, O.; Pedraza-Chaverri, J.; Santana, M.; Esquivel-Guadarrama, F.; Castillo, A.; CervantesTorres, J.; Fragoso, G.; Rosas-Salgado, G. Cucumis sativus aqueous fraction inhibits angiotensin II-induced inflammation and oxidative stress in vitro. Nutrients 2018, 10, 276.

(7) Nash, R. J. Novel anti-inflammatory agent. UK Patent, PCT/ GB2012/000768, WO 2013054070A1 2012.

(8) Manning, K. S.; Lynn, D. G.; Shabanowitz, J.; Fellows, L. E.; Singh, M.; Schrire, B. D. A glucuronidase inhibitor from the seeds of Baphia racemosa: Application of fast atom bombardment coupled with collision activated dissociation in natural product structure assignment. J. Chem. Soc., Chem. Commun. 1985, 127-129.

(9) Booth, K. V.; Jenkinson, S. F.; Watkin, D. J.; Sharp, H.; Jones, P. W.; Nash, R. J.; Fleet, G. W. J. (2S,3R,4R,5S)-3,4,5-Trihydroxypipecolic acid dihydrate $[(2 S, 3 R, 4 R, 5 S)-3,4,5$-trihydroxypiperidine-2carboxylic acid dihydrate]. Acta Crystallogr., Sect. E: Struct. Rep. Online 2007, 63, o3783-o3784.

(10) Martínez, R. F.; Jenkinson, S. F.; Nakagawa, S.; Kato, A.; Wormald, M. R.; Fleet, G. W. J.; Hollinshead, J.; Nash, R. J. from Stevia rebaudiana of DMDP acetic acid, a novel iminosugar amino acid: Synthesis and glycosidase inhibition profile of glycine and $\beta$ alanine pyrrolidine amino acids. Amino Acids 2019, 51, 991-998.

(11) Bashyal, B. P.; Fleet, G. W. J.; Chow, H.-F.; Fellows, L. E. The synthesis of polyhydroxylated amino acids from glucuronolactone. Tetrahedron 1987, 43, 415-422.

(12) Nash, R.J.; Azantsa, B. K.G.; Sharp, H.; Shanmugham, V. Effectiveness of a Cucumis sativus extract (CSE) versus glucosamine- 
chondroitin in the management of osteoarthritis: a randomized controlled trial. Clin. Interventions in Aging 2018, 13, 2119-2126.

(13) Kothari, S.; Saravana, M.; Muthusamy, S.; Mozingo, A.; Soni, M. Safety assessment of a standardized cucumber extract (Q-Actin): Oral repeat-dose toxicity and mutagenicity studies. Toxicol. Reports 2018, 5, 1078-1086.

(14) McFarlin, B. K.; Venable, A. S.; Henning, A. L.; Sampson, J. N. B.; Pennel, K.; Vingren, J. L.; Hill, D. W. Reduced ininflammatory and muscle damage biomarkers following oral supplementation with bioavailable curcumin. BBA Clin. 2016, 5, 72-78.

(15) Frey, A. M.; Satur, M. J.; Phansopa, C.; Parker, J. L.; Bradshaw, D.; Parker, J. L.; Stafford, G. P. Evidence for a carbohydrate-binding module (CBM) of Tannerella forsythia NanH sialidase, key to interactions at the host-pathogen interface. Biochem. J. 2018, 475, $1159-1176$.

(16) Sun, J.; Shigemi, H.; Tanaka, Y.; Yamauchi, T.; Ueda, T.; Iwasaki, $\mathrm{H}$. Tetracyclines downregulate the production of LPSinduced cytokines and chemokines in THP-1 cells via ERK, p38, and nuclear factor- $\kappa \mathrm{B}$ signaling pathways. Biochem. Biophys. Rep. 2015, 4, 397-404.

(17) Nash, R. J.; Kato, A.; Yu, C.-Y.; Fleet, G. W. Iminosugars as therapeutic agents: recent advances and promising trends. Future Med. Chem. 2011, 3, 1513-1521.

(18) Wilson, F. X.; Nash, R. J.; Horne, G.; Storer, R.; Tinsley, J. M. Compounds for the treatment of flaviviral infections. Patent PCT Int. Appl. WO 2010015815A2, 2010.

(19) Watson, A. A.; Fleet, G. W. J.; Asano, N.; Molyneux, R. J.; Nash, R. J. Polyhydroxylated alkaloids - natural occurrence and therapeutic applications. Phytochem 2001, 56, 265-295.

(20) Camussi, G.; Lupia, E. The future role of antitumour necrosis factor (TNF) products in the treatment of rheumatoid arthritis. Drug 1998, 55, 613-620.

(21) Potvin, D. M.; Metzger, D. W.; Lee, W. T.; Collins, D. N.; Ramsingh, A. I. Exogenous interleukin-12 protects against lethal infection with coxsackievirus B4. J. Virol. 2003, 77, 8272-8279.

(22) Romani, L.; Puccetti, P.; Bistoni, F. Interleukin-12 in infectious diseases. Clin. Microbiol. Rev. 1997, 10, 611-636.

(23) Chang, Y.-C.; Uchiyama, S.; Varki, A.; Nizet, V. Leukocyte inflammatory responses provoked by pneumococcal sialidase. mBio 2012, 3, No. e00220.

(24) Katoh, S.; Miyagi, T.; Taniguchi, H.; Matsubar, Y.; Kadota, J. Cutting edge: an inducible sialidase regulates the hyaluronic acid binding ability of CD44-bearing human monocytes. J. Immunol. 1999, 162, 5058-5061.

(25) Jordan, A. R.; Racine, R. R.; Hennig, M. J. P.; Lokeshwar, V. B. The role of CD44 in disease pathophysiology and targeted treatment. Front. Immunol. 2015, 6, 182-196.

(26) Kryworuchko, M.; Gee, K.; Diaz-Mitoma, F.; Kumar, A. Regulation of CD44-hyaluronan interactions in Burkitt's lymphoma and Epstein-Barr virus-transformed lymphoblastoid B cells by PMA and interleukin-4. Cell. Immunol. 1999, 194, 54-66.

(27) Nandi, A.; Estess, P.; Siegelman, M. H. Hyaluronan anchoring and regulation on the surface of vascular endothelial cells is mediated through the functionally active form of CD44. J. Biol. Chem. 2000, $275,14939-14948$.

(28) Gee, K.; Kozlowski, M.; Kumar, A. Tumor necrosis factor-alpha induces functionally active hyaluronan-adhesive CD44 by activating sialidase through p38 mitogen-activated protein kinase in lipopolysaccharide-stimulated human monocytic cells. J. Biol. Chem. 2003, 278, 37275-37287.

(29) Chen, G.-Y.; Chen, X.; King, S.; Cavassani, K. A.; Cheng, J.; Zheng, X.; Cao, H.; Yu, H.; Qu, J.; Fang, D.; Wu, W.; Bai, X.-F.; Liu, J.-Q.; Woodiga, S. A.; Chen, C.; Sun, L.; Hogaboam, C. M.; Kunkel, S. L.; Zheng, P.; Liu, Y. Amelioration of sepsis by inhibiting sialidasemediated disruption of the CD24-SiglecG interaction. Nat. Biotechnol. 2011, 29, 428-435.

(30) Beutler, B.; Cerami, A. The biology of cachectin/TNF - A primary mediator of the host response. Annu. Rev. Immunol. 1989, 7, 625-655.
(31) Burger, D.; Dayer, J.-M. Cytokines, acute-phase proteins, and hormones: IL-1 and TNF-alpha production in contact-mediated activation of monocytes by T lymphocytes. Ann. N.Y. Acad. Sci. 2002, 966, 464-473.

(32) Kaminska, B. MAPK signalling pathways as molecular targets for anti-inflammatory therapy-from molecular mechanisms to therapeutic benefits. Biochim. Biophys. Acta 2005, 1754, 253-262.

(33) Buchholz, K. R.; Stephens, R. S. The extracellular signalregulated kinase/mitogen-activated protein kinase pathway induces the inflammatory factor interleukin- 8 following Chlamydia trachomatis infection. Infect. Immun. 2007, 75, 5924-5929.

(34) Baldassare, J. J.; Bi, Y.; Bellone, C. J. The role of p38 mitogenactivated protein kinase in IL-1b. transcription. J. Immunol. 1999, 162, 5367-5373.

(35) Ahr, H. J.; Boberg, M.; Brendel, E.; Krause, H. P.; Steinke, W. Pharmacokinetics of miglitol. Absorption, distribution, metabolism, and excretion following administration to rats, dogs, and man. Arzneimittelforschung 1997, 47, 734-745.

(36) Takasu, S.; Parida, I. S.; Onose, S.; Ito, J.; Ikeda, R.; Yamagishi, K.; Higuchi, O.; Higuchi, F.; Kimura, T.; Miyazawa, T.; Nakagawa, K. Evaluation of the anti-hyperglycemic effect and safety of microorganism 1-deoxynojirimycin. PLoS One 2018, 13, No. e0199057.

(37) Amezqueta, S.; Ramos-Romero, S.; Martínez-Guimet, C.; Moreno, A.; Hereu, M.; Torres, J. L. Fate of D-fagomine after oral administration to rats. J. Agric. Food Chem. 2017, 65, 4414-4420.

(38) Yang, S.; Mi, J.; Liu, Z.; Wang, B.; Xia, X.; Wang, R.; Liu, Y.; Li, Y. Pharmacokinetics, , tissue distribution, and elimination of three active alkaloids in rats after oral administration of the effective fraction of alkaloids from Ramulus mori, an innovative hypoglycemic agent. Molecules 2017, 22, 1616.

(39) van Schouwenburg, P. A.; Rispens, T.; Wolbink, G. J. Immunogenicity of anti-TNF therapies for rheumatoid therapies for rheumatoid arthritis. Nat. Rev. Rheumatol. 2013, 9, 164-172.

(40) Waljee, A. K.; Rogers, M. A. M.; Lin, P.; Singal, A. G.; Stein, J. D.; Marks, R. M.; Ayanian, J. Z.; Nallamothu, B. K. Short term use of oral corticosteroids and related harms among adults in the United States: population based cohort study. Br. Med. J. 2017, 357, j1415.

(41) Ong, C. K. S.; Lirk, P.; Tan, C. H.; Seymour, R. A. An evidencebased update on nonsteroidal anti-inflammatory drugs. Clin. Med. Res. 2007, 5, 19-34. 